\title{
Igualdad, derecho y capital: Una lectura crítica
}

Equality, law and capital: A critical reading

Igualdade, Direito e Capital: Uma leitura crítica

Égalité, droit et capital: Une lecture critique

平等, 法律与资本:批判性阅读

Pablo Gres Chávez ${ }^{1}$ | Universidad Católica del Norte, Chile

Revista Derechos en Acción ISSN 2525-1678/ e-ISSN 2525-1686

Año 4/N 13, Primavera 2019 (21 septiembre a 20 diciembre), 271-303

DOI: https://doi.org/10.24215/25251678e338

ORCID: https://orcid.org/0000-0002-7202-957X

Recibido: 23/10/2019

Aprobado: 28/11/2019

Resumen: El trabajo presente tiene como objetivo principal estudiar la relación entre derecho, igualdad, y capital. Se estructura atendiendo al siguiente orden. En primer lugar, presenta una propuesta metodológica de análisis jurídico desde el materialismo histórico. En segundo lugar y atendiendo a dicho método, se estudia la relación entre sujeto jurídico, ciudadanía, igualdad y Derecho. En tercer término, busca develar una contradicción fundamental inmanente a la forma jurídica, dada por la relación entre los conceptos antes abordados. Finalmente ofrece algunas conclusiones relacionadas con el pensamiento de Marx, la solidaridad y el Derecho.

Palabras clave: Marxismo - Capital - Derecho - Igualdad - Contradicción.

Abstract: The purpose of this paper is to study the relationship between Law, equality, and capital. It is structured according to the following order. First, it presents a methodological proposal of legal analysis from

\footnotetext{
1 Licenciado en Ciencias Jurídicas y Sociales, U. de Chile. Doctor (C) en Derecho, U. de Buenos Aires. Profesor de Filosofía del Derecho, U. Católica del Norte, sede Coquimbo, Chile. Correo: pablo.gres@ucn.cl.
} 
historical materialism. Secondly and according to this method, the relationship between legal subject, citizenship, equality and law is studied. Third, it seeks to reveal a fundamental contradiction immanent to the legal form, based on the relationship between the concepts previously studied. Finally, it offers some conclusions related to Marx's thinking, solidarity and law.

Keywords: Marxism - Capital - Law - Equality - Contradiction.

Resumo: 0 presente trabalho tem como objetivo principal estudar a relação entre lei, igualdade e capital. Está estruturado de acordo com a seguinte ordem. Primeiro, apresenta uma proposta metodológica de análise jurídica a partir do materialismo histórico. Em segundo lugar e de acordo com esse método, estuda-se a relação entre sujeito legal, cidadania, igualdade e direito. Terceiro, procura revelar uma contradição fundamental imanente à forma jurídica, dada pela relação entre os conceitos antes discutidos. Por fim oferece algumas conclusões relacionadas com o pensamento de Marx, a solidariedade e o direito.

Palavras-chave: Marxismo - Capital - Direito - Igualdade - Contradição

Résumé: Le présent travail a pour objectif principal d'étudier la relation entre le droit, l'égalité et le capital. II est structuré selon l'ordre suivant. Tout d'abord, il présente une proposition méthodologique d'analyse juridique à partir matérialisme historique. En second lieu et conformément à cette méthode, il analyse la relation entre sujet juridique, citoyenneté, égalité et droit. Troisièmement, il cherche à révéler une contradiction fondamentale immanente à la forme juridique, étant donné les relations entre les concepts évoqués ci-dessus. Enfin, il offre quelques conclusions liées à la pensée de Marx, à la solidarité et au droit.

Mot-clés: Marxisme - Capital - Droit - Egualité - Contradiction.

摘要: 本研究的主要目标是研究法律, 平等与资本之间的关系。它是 按照以下顺序构造的。首先, 它提出了从历史唯物主义角度进行法 律分析的方法论建议。其次, 根据这种方法, 研究了法律主体, 公民 身份, 平等与法律之间的关系。第三, 它试图揭示上述概念之间的关 系所给定的法律形式固有的基本矛盾。最后, 它提供了与马克思的 思想, 团结和法律有关的一些结论。

关键字: 马克思主义,资本, 法律, 平等-矛盾。 


\section{Introducción}

La presente investigación, tiene como objetivo principal investigar la relación que existe entre el Derecho y la igualdad, utilizando como marco teórico la tradición del materialismo histórico.

El marxismo ha sostenido que el capital, en particular, y la formación social capitalista, en general, están llenos de relaciones contradictorias. Destacan la relación capital-trabajo, valor de uso-valor de cambio o Estado-propiedad privada, todas ellas relaciones contradictorias. Para Marx, "en la producción capitalista todo es contradictorio y se manifiesta bajo una forma antagónica" ${ }^{2}$.

La presente investigación se desarrolla teniendo presente aquella premisa. Pretende estudiar la relación entre igualdad, capital y Derecho, teniendo en consideración el carácter intrínsecamente contradictorio del modo de producción actual. En este sentido, el Derecho es concebido desde el marxismo como una relación social que, por un lado, promete igualdad y, por otro, genera las condiciones para perpetuar la desigualdad.

Sostenemos, como primera hipótesis de trabajo, que el Derecho ofrece un horizonte valorativo igualitario. Esta hipótesis, está íntimamente arraigada en una comprensión del fenómeno jurídico que sostiene que éste no puede ser estudiado sino en relación a la modernidad, como periodo histórico determinado y al modo de producción que da origen a aquel periodo. Nuestra segunda hipótesis, ofrece una perspectiva crítica en torno a la relación entre igualdad, capital y Derecho. Sostenemos que éste no puede cumplir aquel horizonte igualitario ya que en él (y en toda la formación social a la que responde) yace una contradicción de carácter fundamental.

En la primera sección de la investigación, ofreceremos una perspectiva metodológica ligada a la teoría jurídica crítica (histórico-materialista) que nos habilita a llevar a cabo un

2 Marx, Karl. (1980). Historia crítica de la Teoría de la Plusvalía (T1). FCE. México, p. 199. 
análisis más allá de lo meramente normativo. Precisamente, una de las ventajas que ofrecen las teorías críticas, es que "producen una ruptura de carácter epistemológico porque abandonan un modelo explicativo y lo sustituyen por un modelo dialécticocomprensivo"3. Para este respecto, la perspectiva ofrecida por el jurista E. B. Pashukanis será fundamental.

La segunda sección, examinara el contenido igualitario presente en el Derecho en tanto producción moderna. Será fundamental el análisis de las categorías de sujeto de derecho o sujeto jurídico y la categoría de ciudadano.

La tercera sección estudiará la contradicción que yace en el núcleo del de la formación jurídica moderna. Para esto volveremos a analizar las nociones de sujeto jurídico y ciudadano.

Finalmente, ofreceremos algunas luces, a modo de colofón, que el pensamiento de Marx ofrece para solucionar el problema planteado.

\section{Marxismo y Derecho: El derecho como relación social}

Desde una perspectiva histórico-materialista, el derecho puede ser concebido como una relación social históricamente determinada. Desde el punto de vista marxista, las relaciones sociales atienden a conductas o prácticas de individuos en relación a otros, estas prácticas están, de uno u otro modo determinadas por el momento histórico en que se desarrollan, pero a su vez la humanidad tiene una capacidad transformadora que le permite avanzar.

En tal sentido, el materialismo histórico no otorga al derecho el estatus de una estructura que goce de autonomía absoluta. Más bien, es parte de un conjunto de relaciones sociales del que no puede ser despojado porque, precisamente, lo determinan.

Desde esta tradición la historia es comprendida como un conjunto de relaciones sociales internamente imbricadas,

3 Ruiz, Alicia E. C. (2000). Idas y vueltas: por una teoría crítica del Derecho. Buenos Aires. UBA, p. 4. 
determinadas por relaciones sociales anteriores. Éstas no son estáticas sino más bien dinámicas. En otros términos, la historia es negatividad pura, todo momento contiene la posibilidad de su negación, o su superación. En términos prácticos, esta posibilidad de negación y superación está en el seno de las relaciones entre las distintas clases, una clase históricamente se ha ocupado de revolucionar los medios de producción con el objeto de apropiar cada vez más valor de la producción social; la otra, busca precisamente recuperar ese valor que considera usurpado.

Esta perspectiva, que pone a la humanidad en el centro de su propia historia, no es otra cosa que la proposición de un bumanismo absoluto. "Una premisa ontológica que no admite exterior natural alguno, que requiere pensar toda diferencia como diferencia interna. Una premisa que puede llamarse, propiamente, humanismo absoluto" ${ }^{4}$. De este modo, la humanidad sólo responde a su propio cause histórico. No hay naturaleza humana alguna.

El historicismo absoluto formulado por el marxismo, le permite sortear toda objeción que reconozca a la bondad o a la maldad o a cualquier otra objeción de corte apriorístico o naturalista, como característica determinante de lo humano. Si no hay naturaleza humana, no hay cabida para el naturalismo jurídico.

Por otro lado, si el Derecho es parte del conjunto de relaciones sociales que conforman la formación económico-social capitalista, carece de sentido estudiar el derecho como un objeto separado de las otras relaciones que mantienen los sujetos, tal y como el positivismo jurídico ha propuesto.

Sostenemos que hay dos principales objeciones contra el positivismo. En primer término, creemos que el positivismo jurídico (sobre todo el de orden kelseniano) adolece de un grave

4 Pérez Soto, Carlos. (2001). Para una crítica del poder burocrático: comunistas otra vez. Santiago de Chile. LOM-U. ARCIS., p 58. 
problema epistemológico: pone como condición de posibilidad para el sistema jurídico, la norma hipotética fundamental.

En un primer momento, Kelsen sostiene que la norma hipotética fundante es una norma, de naturaleza hipotética, pero una norma al fin y al cabo. Todo el derecho adquiere su validez, en el sistema dinámico, de aquella norma. Pero ocurre que si la norma fundante básica es hipotética, su validez no puede más que suponerse. Es decir si nos, "ceñi[mos] con rigor al pensamiento kelseniano, no hay que hablar de derecho válido sino del derecho supuesto válido inmediata o mediatamente por la norma básica"'.

En sus distintas formulaciones, Kelsen optó por proponer una teoría del derecho que separa radicalmente al sujeto observador, del objeto observado. La premisa básica de la observación científica, es que el juicio del observador debe restringirse a realizar una mera descripción de aquello que precisamente se observa. Si Kelsen cierra el sistema jurídico con una norma hipotética, lo que hace no es describir un hecho (tarea del científico observador y descriptor), sino prescribir una forma en que debe ser observado un hecho. La condición de posibilidad kelseniana, en la que descansa su sistema, no tiene fundamento epistemológico alguno más que un cierre antojadizo del objeto observado, por parte del sujeto observador.

El segundo argumento se relaciona con un giro en el argumento kelseniano. La norma hipotética pasa de ser una norma a ser una ficción al estilo del neokantiano Vehinger ${ }^{6}$. Pero, tal y como ha sostenido Carlos Cárcova,

"si el fundamento del orden es una ficción, la tesis de que el ejercicio del poder se legitima en la sociedad occidental mediante la movilización del imaginario social, esto

\footnotetext{
5 Macía Manso, Ramón. (2012). Cientificismo. Modalidades. Consecuencias. Oviedo, España. Ediciones de la U. de Oviedo. p. 196. Los corchetes son adecuación nuestra.

6 Cfr.: Kelsen, Hans. (2010). "La función de la constitución”, en: Revista del Instituto de investigación Ambrosio L. Gioja (año IV, nº 5). Pp. 150-157.
} 
es, mediante la operatividad del discurso de la ideología, retoma la importancia crítica que muchos pensadores le han atribuido"7.

La forma en que Kelsen cierra el sistema inevitablemente abre otros flancos que, al menos, incomodan a las pretensiones del positivismo. Tal y como sostuvo Manuel Sacristán (en un interesante aunque incompleto trabajo), "la función apologética del positivismo jurídico, su naturaleza de ideología burguesa no se manifiesta en la interioridad cerrada del sistema científicojurídico [...] sino en el plano filosófico más general"8.

La perspectiva metodológica para el análisis del Derecho que ofrece el materialismo histórico es radicalmente distinta a la naturalista y a la iuspositivista. Esta forma de comprender el fenómeno jurídico, sostiene que éste no tiene autonomía absoluta. Es filosófica y epistemológicamente inviable sostener aquello. Carece de sentido estudiar el Derecho desde su autoproducción, o dicho de otro modo, éste "no puede entenderse a partir de sus propios enunciados lógicos-abstractos".

En lo que refiriere al método, el primer teórico marxista que mejores herramientas ofreció para el estudio del derecho es Evgeny Branislav Pashukanis. En 1924 escribió la obra que fundó las bases para el estudio del Derecho desde la perspectiva marxista ${ }^{10}$. El aporte teórico de Pashukanis es contundente, tanto para la filosofía del Derecho, como para la teoría crítica del Derecho.

7 Cárcova, Carlos. (2012). Las Teorías Jurídicas Post Positivistas. Buenos Aires. AbeledoPerrot, p. 9

8 Sacristán, Manuel. (1984). “De la idealidad en el Derecho”. En: Sacristán, Manuel. 1984. Papeles de filosofía. Panfletos y materiales II. Icaria Antrazyt. España, p. 315

9 Rivera Lugo, Carlos. (2017). “Comunismo y Derecho: reflexiones sobre la crisis actual de la forma jurídica", en Correas, Oscar y Wolkmer, Antonio. 2017. La teoría crítica del Derecho en América latina. España. Akal. p. 699.

10 Cfr.: Pashukanis, E. B. (1976). La teoría general del Derecho y el marxismo. Barcelona, Grijalbo. 
Si bien su obra ya ha cumplido casi cien años, su esfuerzo teórico y particularmente su propuesta metodológica, han sido rescatados y reinterpretados por el marxismo contemporáneo.

Metodológicamente, Pashukanis se aparta de la filosofía jurídica imperante y utiliza la abstracción como propuesta inicial. Se propone estudiar y desarrollar los conceptos jurídicos más básicos y fundamentales, tales como norma jurídica, relación jurídica o sujeto jurídico. Sostiene que el concepto jurídico básico en el Derecho moderno, es decir, en el Derecho que responde a la formación social capitalista, es el de sujeto jurídico. Su propuesta es interesante en varios sentidos.

En primer término, es coherente con el método utilizado por Marx, quien comienza el libro I de su obra más importante, El Capital, con el análisis de la mercancía, como unidad básica de la economía política. "El hombre llega a ser sujeto jurídico por la misma necesidad por la cual se transforma el producto natural en una mercancía dotada de la enigmática propiedad de valor" ${ }^{\prime 1}$. En segundo lugar, no es una posición metodológica normativista. Para el autor, las normas no tienen sentido sin sujetos que las materialicen. En tercer lugar, si el Derecho es una relación social institucionalizada (o fetichizada), tiene pleno sentido que su unidad básica sea el sujeto, ya que las relaciones sociales precisamente se constituyen entre sujetos.

La formación económico-social capitalista es, en esencia, una forma de organizar el mundo en el que son claves: la transmutación de trabajo en capital y el intercambio de mercancías. A diferencia del periodo inmediatamente anterior a la modernidad, el intercambio de mercancías entre individuos era inconcebible. La modernidad abre paso a esta posibilidad de intercambio. El mercado define a la modernidad y al capitalismo.

Para Pashukanis, todo intercambio se hace mediante un acto jurídico. La relación de intercambio, es una relación general,

11 Pashukanis, E. B. (1976). La teoría general del Derecho y el marxismo. Barcelona, Grijalbo.
p. 55. 
indeterminada, que no refiere a individuos particulares sino que existe como posibilidad para todos aquellos que deseen llevarla a cabo. Es por esa razón que la forma jurídica no regula la relación de intercambio entre sujetos determinados, sino que entre sujetos abstractos, generales, libres e iguales formalmente.

Pashukanis sostiene que, "al mismo tiempo en que el producto del trabajo se transforma en una mercancía y así en portadora de valor, los hombres adquieren la capacidad de ser sujetos de derecho" ${ }^{12}$. Todos los hombres y mujeres, son formalmente iguales en tanto poseedores de mercancías o, más bien, todos pueden ser potencialmente considerados como tales.

La reflexión de Pashukanis es notable, ya que desde su posición, el contendido valorativo meta-jurídico, expresado en algunos de los primeros y más importantes textos político-normativos modernos (como la Declaración de independencia de los Estados Unidos de 1776 o la Declaración de los derechos del hombre y del ciudadano de 1789), no sería abstracto, vacío de contenido material; sino que estos valores, o más bien su contenido cierto, serían un producto histórico, íntimamente relacionado a la formación social capitalista. Estarían, por decirlo de otro modo, históricamente determinados. Así, Pashukanis anuncia una reinterpretación respecto de la determinación entre estructura económica y superestructura jurídico-política.

Una visión muy simplista extraída de una serie de citas localizadas de Marx, ha sostenido que la base económica determina la superestructura jurídico-política de forma unidireccional. Desde esta perspectiva todo el derecho es concebido como un beneficio para la clase dominante. La teoría de Pashukanis es absolutamente mucho más minuciosa y sugerente.

En primer lugar, su propuesta es innovadora ya que para él, la forma jurídica es parte de la base económica. Todo intercambio de mercancía es investido por la forma jurídica, y se realiza entre sujetos jurídicos. El producto material de esta

12 Ibíd. p. 112 
relación social, es traducido en normas que son funcionales al intercambio y a la formación social capitalista. Según Mieville, puede entenderse que en Pashukanis, son las normas las que son parte de la superestructura (jurídico política), no todo el derecho. En palabras del autor "la forma jurídica es parte de la base, y se actualiza a través de las particularidades necesarias de la superestructura legal"13.

Desde esta perspectiva, estructura y superestructura no son más que categorías analíticas y no es posible realizar una separación real de las mismas. Es más, no es viable delimitar de forma clara los bordes de cada una de aquellas esferas. Así, Economía, Derecho y política son parte de un todo orgánico. La formación económico-social capitalista debe ser considerada una unidad orgánica. "Es necesario asumir un punto de partida radicalmente distinto al que subyace en las operaciones actuales: la vida en sociedad constituye una unidad, con dimensiones económicas, políticas y sociales, y es desde ella donde se puede hacer comprensible" ${ }^{14}$.

Las normas así entendidas, no pueden ser miradas de forma aislada. Su forma y sobre todo su contenido permiten la actualización de la base económica. El contenido de las normas jurídicas es siempre político, entendiendo por política la organización del poder. La relación tensa y contradictoria entre las clases sociales, permea necesariamente el contenido de las normas jurídicas.

La formación social capitalista necesita de sujetos indeterminados, iguales y abstractos. A su vez, como sostiene Juan Ramón Capella "a partir de condiciones económicas que suponen la explotación de unas personas por otras surgió un mecanismo

\footnotetext{
13 Mieville, China. (2005). Between equal rights: A marxist theory of Internacional Law. Brill: Oxford. Inglaterra. p. 96. Traducción propia. La cita en inglés es la siguiente: So the legal form is of the base, and it actualises through the necessary particularities of the legal superstructure. 14 Osorio, Jaime. (2014). Estado, reproducción del capital y lucha de clases: la unidad económico/política del capital. U. Autónoma de México - Instituto de investigaciones económicas-CLACSO. México. p. 13.
} 
cohesionante necesario, al que de momento llamamos el poder (de gobierno/militar/cultural), que asegura la reproducción social en esas condiciones"15. Un asunto clave, es que en términos reales, concretos y materiales, algunos individuos no poseen otra mercancía más que ofrecer que su fuerza de trabajo (son desiguales materialmente) y deben ponerla a disposición de aquellos que poseen medios de producción, para producir las mercancías, que estos pondrán en el mercado. "No existe ninguna relación económica que no tenga por forma una relación jurídica o política. De manera correspondiente, no existe ninguna relación jurídica o política que no tenga por contenido la organización indirecta del trabajo social”"16.

La relación tensa entre igualdad y explotación en relación al Derecho ocupará nuestro análisis en las próximas páginas.

\section{Igualdad y Derecho}

\section{a. Modernidad e Igualdad}

En la sección anterior, hemos adelantado que el Derecho moderno ofrece un horizonte igualitario. Es más, sostenemos que la igualdad es parte de su núcleo. Los sujetos de derecho son, por antonomasia, sujetos iguales. De esta forma, en el discurso jurídico, "la igualdad aparece como un grundwert, como un valor-guía de la ética política, jurídica y social" ${ }^{17}$.

La modernidad, en cuanto periodo histórico, se caracteriza por ser el momento en que comienzan a operar relaciones sociales completamente distintas a aquellas que regían en el antiguo régimen. La modernidad es el periodo histórico en que

\footnotetext{
15 Capella, Juan Ramón. (2008). Fruta prohibida: una aproximación histórico-teorética del derecho y del Estado. España. Trotta. p. 67.

16 Carrera, Juan Îñigo. (2012). "El capital: determinación económica y subjetividad política": en: Crítica Jurídica (n³4). UNAM: México p.64.

17 Pérez Luño, Antonio. (2007). Dimensiones de la igualdad. Cuadernos "Bartolomé de las Casas" (núm. 34). Dykinson. Madrid. p. 15.
} 
se posiciona como dominante aquel entramado complejo de relaciones sociales que el marxismo y particularmente la historiografía ha llamado capitalismo.

La modernidad implica cambios radicales respecto del antiguo régimen.

"Más aún, tales cambios implican un modo radicalmente nuevo de vivir en comparación con las formaciones sociales precapitalistas: con respecto, por ejemplo, a la relación activa y transformadora entre el hombre y la naturaleza característica del capitalismo, al desarrollo de formas de vida urbana cualitativamente nuevas y al surgimiento de una concepción del tiempo lineal y homogénea"18.

Para el materialismo histórico, el ordenamiento jurídico moderno es producto de ese entramado complejo de relaciones sociales que hemos denominado capitalismo. Al no concebirse el Derecho como una disciplina aséptica y neutra, se puede reconocer que éste tiene, al menos en un sentido mínimo, un contenido igualitario al consagrar la igualdad formal de los sujetos jurídicos y yendo un paso más allá, reconociendo la igualdad formal de todos los ciudadanos.

"En tanto adhieren a la idea de igualdad ante la ley o, en un plano más abstracto, a la idea de igualdad moral, de acuerdo con la cual todos los seres humanos están investidos de la misma dignidad y son pasibles del mismo respeto por parte del Estado"19.

Todo ordenamiento jurídico moderno, considera como iguales a sus ciudadanos, a lo menos, en los simple términos señalados. Esta mínima consideración es una muestra de dos asuntos de importancia. En primer lugar, da cuenta de una de las características propias de la burguesía como clase social, y es que revoluciona constantemente los medios de producción y transforma las relaciones de producción (y obviamente su

18 Callinicos, Alex. (2011). Contra el posmodernismo. Buenos Aires. Ediciones RyR, pp. 98-99.

19 Alegre, Marcelo. (2010). Igualdad, Derecho y Política. Buenos Aires. Fontamara. P. 13. 
control). Es en este sentido que Marx sostuvo que "la burguesía ha desempeñado papel eminentemente revolucionario en la historia" ${ }^{20}$. En segundo lugar, al ser una conquista burguesa, el sistema jurídico moderno puede ser considerado burgués, en esos términos, no como una creación de la mala voluntad de la clase dominante para oprimir a la clase explotada.

Con la modernidad, los individuos reales y sus condiciones materiales, desaparecen en el momento en que el Derecho toma parte de sus vidas. Esto ocurre de dos maneras. La primera es a través la igualación formal mediante la conversión del individuo determinado en sujeto jurídico general y abstracto; y la segunda, es mediante la conversión del individuo en ciudadano abstracto de un Estado.

En tanto sujetos de derecho o ciudadanos de Estado, los individuos no son considerados en su rol en la división social del trabajo, sino que por el contrario, como sujetos de derecho o como ciudadanos formales toda desigualdad desaparece. Incluso, el ciudadano en cuanto individuo, y sin considerar la clase a la que pertenece, se sumerge en un proyecto de espíritu común, fraterno y homogeneizador, el del pueblo, la sociedad civil, el de la nación o el del Estado.

Pero, las condiciones históricas y materiales de la humanidad, han hecho de la igualdad una verdadera consigna de lucha permanente, una especie de horizonte valorativo a alcanzar. La igualdad aspira ser materializada, sin estar del todo claro cuál es su contenido.

"Desde que hay leyes y constituciones, desde que el ejercicio del poder debe legitimarse como fundado en razón y en derecho y no como un simple estado de hecho, ese poder debe situar en alguna parte esa igualdad que lo funda y lo disuelve al mismo tiempo, que lo legitima y lo deslegitima"21.

\footnotetext{
20 Marx, Karl. (1971). Manifiesto comunista. Editorial Universitaria. Santiago, p. 9.

21 Rancière, Jacques. (2007). El método de la igualdad, conferencia en Cerisy-La Salle. Francia
} 
En tanto relación social entre sujetos iguales el Derecho moderno (en relación a la igualdad), es siempre plural, relacional y permite la comparación. La primera dimensión de la igualdad, analíticamente hablando, es la igualdad formal que se expresa como igualdad ante la ley. Todos y cada uno de los individuos son regidos por un mismo estatuto jurídico, lo que garantiza la igualdad de trato. Así, el derecho moderno reconoce la igualdad como un principio rector, deudor de la isonomía griega o de la aequitas romana. "En la lucha contra la arbitrariedad y el despotismo las revoluciones burguesas del XVIII proclamaron la igualdad jurídica de todos los hombres"22.

La igual consideración de todos los hombres, o más preciso aún, de los ciudadanos, condiciona de forma determinante la manera en que se erige el derecho. Dicho de otro modo, hay una relación latente e indisoluble entre el principio de soberanía popular y la igualdad formal. Aquel, proclama que el único sujeto legislador y soberano es el pueblo, entendiendo por éste el conjunto de todos sus ciudadanos, nadie es considerado mejor, ni privilegiado, sino como mero miembro del pueblo.

De esta forma, la ley (y también la Constitución), es el instrumento mediante el que el pueblo hace ejercicio de su soberanía. En el relato liberal, el señor soberano del antiguo régimen es reemplazado, y su poder se diluye entre todos y cada uno de los miembros del pueblo, los ciudadanos; y como conjunto eleva a la ley (encarnada en la Constitución) al lugar que antes ocupó el señor. El Derecho, desde esta perspectiva, es producto de la voluntad general. El sistema jurídico y obviamente también el Estado, es una herramienta de y para sujetos libres e iguales.

Esto no puede ser de otra forma. Los sujetos libres e iguales no sólo participan de la política, sino que también del mercado. Ni la política, ni el Derecho, ni el mercado pueden sobrevivir sin las demás esferas. En el mercado, lo que se intercambia son mercancías consideradas equivalentes, ese intercambio sólo

22 Pérez Luño. Óp. Cit, p.19. 
puede hacerse entre sujetos (jurídicos) iguales. Aquella consideración es claramente política. Sólo entre iguales puede considerarse que se realiza un intercambio de (mercancías) equivalentes. Para eso, un ordenamiento general y abstracto, es imprescindible.

La igualdad en su dimensión formal, es en un sentido mínimo una exigencia de generalidad. Un sistema normativo general y abstracto que considere a los individuos en su calidad de sujetos de derecho es una premisa básica del constitucionalismo moderno. Los privilegios o inmunidades, prácticamente estáticos y naturalizados propios de la formación social anterior, desaparecen en el momento en que la ley no se dirige a ningún sujeto en particular.

Lo anterior puede tener una segunda lectura. Si la ley se considera general, implica que se exige un trato igual para lo que se considera igual. Pero desde un mero ejercicio lógico, puede concluirse que la exigencia de generalidad es, a su vez, una exigencia de equiparación. Es decir, se consideran iguales sujetos en condiciones materiales desiguales, pero aquellas desigualdades se consideran irrelevantes. Esto, en un sentido restringido.

La exigencia de equiparación también es, en un sentido más amplio, la puerta de entrada para otros criterios valorativos ligados a la igualdad.

"De ahí, que la evidencia de que los hombres no son jamás enteramente iguales haya obligado a los defensores históricos del valor de la igualdad a hacer abstracción (que no significa negación) de los rasgos diferenciales caracterizadores de las distintas personalidades, para insistir en la igualdad fundamental de su dignidad (Santo Tomás), de su racionalidad (Locke, Kant), de sus pasiones (Bentham, Mill) o de su pertenencia a la esencia genérica humana (Marx)"23.

23 Ibíd. p. 25 
Con esto, vemos que poco a poco la idea simple de igualdad formal, se nutre cada vez más de un contenido material.

A estas alturas, cabe preguntarse ¿cómo la igualdad formal puede hacerse cargo de situaciones desiguales que no pueden ser ignoradas como la desigualdad de estrato o de ingreso? Sostenemos que una tercera exigencia de la igualdad formal, es la de diferenciación. Así, en algunos casos, el derecho no es ciego a las condiciones materiales de los individuos, sin llegar a hacerse cargo de los individuos propiamente tal. Un buen ejemplo de esto es la incorporación de la progresividad en la legislación tributaria.

Hemos venido sosteniendo que la igualdad ante la ley, como mera dimensión formal de la igualdad, tiene que enfrentarse constantemente con las condiciones generadas por la desigualdad material. Esto, ya que la igualdad no es solo un valor, sino que también es una condición política que estructura la forma jurídica. "Como demanda social y política concreta, la igualdad es hija de las grandes revoluciones que inauguraron el mundo moderno" 24 . La modernidad se ha estructurado atendiendo a una constante lucha por la igualdad y la libertad.

"La aspiración a la igualdad fue uno de los ideales constitutivos de la modernidad capitalista desde el momento en que triunfaron las grandes revoluciones burguesas" ${ }^{25}$ y sigue siendo eso: una aspiración. Aquello, no es precisamente un buen síntoma, sino que es más bien una demostración de que la modernidad ha fracasado en su tarea. Cabe preguntarse, entonces, cuál es el problema de fondo filosóficamente hablando. Nadie podría negar, que el Derecho moderno ofrece un enorme avance en términos igualitarios, al ser la herramienta que sentencia a muerte el orden jerárquico del antiguo régimen. Entonces, ¿qué es lo que va mal?

\footnotetext{
24 Callinicos, Alex. (2006). Igualdad. Siglo XXI. España. p. 33

25 Callinicos, Alex. (2003). “Igualdad y capitalismo”, en: Borón, Atilio. 2003. La teoría Marxista hoy. CLACSO. Argentina.
} 
En una reciente publicación en castellano, el filósofo francés Étienne Balibar, ha insistido en la necesidad de reconocer que igualdad y libertad no atienden a cuestiones distintas, muy por el contrario, se debe entender que la extensión material de ambas es idéntica (esto no significa que sean lo mismo). Balibar, nutriendo su argumento, ha acuñado el concepto compuesto de igualibertad, "planteando que la negación de la libertad destruye de hecho la igualdad, y que la negación de la igualdad destruye de hecho la libertad. Precisamente debido a eso es imposible escoger una contra otra" ${ }^{26}$. El argumento de Balibar es político y a su vez jurídico. Es político porque el reconocimiento de la igualdad es siempre un límite al poder; y es jurídico, porque el derecho es la forma en que el poder ha visto su límite.

\section{b. Igualdad, Capital y Derecho}

El contenido de la igualdad en tanto derecho no está del todo claro. Lo que sí es cierto, recalca Callinicos, es que

"las reivindicaciones políticas de las grandes revoluciones de los siglos XVII y XVIII tienen una tendencia inherente a rebasarse a sí mismas por sus flancos. Ideales a los que inicialmente se atribuía una referencia bastante estrecha, cuyo fin era beneficiar fundamentalmente a los varones blancos y propietarios, demostraban ser susceptibles de una extensión indefinida. El resultado es un proceso de revolución permanente en el que una sucesión de nuevos sujetos políticos - trabajadores, esclavos, mujeres, súbditos coloniales, gente de color, nacionalidades oprimidas, lesbianas, gays, minusválidos [...] - surgen para plantear su reivindicación a la libertad y a la igualdad que han conquistado luchas anteriores" ${ }^{27}$.

El contenido de la igualdad no es claro, no por su opacidad, sino por la propia historicidad inmanente al concepto.

${ }^{26}$ Balibar, Étienne. (2017). La igualibertad. España. Herder. p.180.

27 Callinicos. (2006). Óp. Cit. p. 37. 
La igualdad coincide con el Derecho, la política y el mercado, porque a la vez los constituye; y son sus tensiones las que actualizarán su contenido.

Nuestro análisis ha comenzado con la categoría de sujeto jurídico ya que es la primera forma en que la igualdad permea el Derecho. En abstracto, todo sujeto de derecho es capaz de intercambiar su propiedad o su fuerza de trabajo en el mercado. Para facilitar aquello, se ha construido todo un sistema normativo que tiene como garante al Estado.

Para participar del mercado, los sujetos de derecho, no sólo deben ser considerados libres e iguales, sino que, obviamente, deben tener las condiciones materiales para poder hacerlo, deben tener algo que intercambiar, en otras palabras, deben tener propiedad o dominio sobre cosas.

Pero ocurre que, aunque la ley considere a todos sus ciudadanos iguales, hay situaciones que los hacen profundamente desiguales. La modernidad ha sido marcada por una diferencia determinante. Hay un grupo de personas que poseen medios de producción que les permite generar mercancías para intercambiar y otro grupo, mayoritario, que no tiene otra mercancía que ofrecer más que su fuerza de trabajo, es decir, su consentimiento para que otro haga uso de aquella fuerza.

Respecto de los derechos fundamentales que están en juego en el mercado, Marx, en su principal obra, ha señalado que

"[l]a órbita de la circulación o del intercambio de mercancías, en cuyo marco se desenvuelve la compra y venta de la fuerza de trabajo, era, en realidad, un verdadero edén de los derechos innatos del hombre. Dentro de sus límites imperan la libertad, la Igualdad, la propiedad y Bentham. ¡La libertad! pues el comprador y el vendedor de una mercancía, por ejemplo la fuerza de trabajo, se someten sólo a su libre voluntad. Contratan como hombres libres e iguales jurídicamente. El contrato es el resultado final en que sus voluntades cobran una expresión jurídica común. ¡La Igualdad!, pues compradores y vendedores se refieren recíprocamente sólo como 
poseedores de mercancías, cambiando equivalente por equivalente. ¡La propiedad!, pues cada uno dispone únicamente de lo que posee. ¡Y Bentham!, pues cada uno se preocupa únicamente de sí mismo"²8

Gran parte de la obra de Marx está destinada a hacer una crítica contundente de la economía política de su tiempo. Una de sus posiciones más importantes (que hace unas líneas adelantamos), sostiene que una grupo minoritario, la clase explotadora o dominante, hace uso de la fuerza de trabajo de otro grupo mayoritario, que sólo posee como mercancía su cuerpo, la clase trabajadora. La clase trabajadora produce mercancías para la clase explotadora, mercancías que tienen como finalidad ser transadas en el mercado. Esta se denomina en la tradición marxista, explotación.

Aquí, surge el primer gran problema de la igualdad en tanto derecho meramente formal. Los sujetos son considerados jurídicamente iguales en abstracto, para poder intercambiar sus mercancías en el mercado. Pero el mercado necesita de sujetos materialmente desiguales que muevan la producción capitalista. Es determinante, entonces, el hecho de que a un grupo mayoritario de la humanidad les sea restringido el acceso a los medios de producción.

Cuando salimos de la esfera del intercambio donde los sujetos son considerados iguales formalmente; cuando

"seguimos al capitalista y al trabajador al domicilio oculto de la producción, la imagen cambia. La igualdad entre ambos es sólo formal; en realidad, son desiguales. [...] La aparente libertad e Igualdad del trabajador respecto al capitalista oculta una subordinación y desigualdad subyacente cuyo resultado es la explotación del primero" ${ }^{29}$.

A su vez, el Derecho y el Estado, ofrecen una categoría abstracta adicional. La calidad de ciudadano. Más bien, la

28 Marx, Karl. (2010). El capital: Crítica de la economía política (T1). Santiago de Chile. LOM. pp. 182-183.

${ }^{29}$ Callinicos, Alex. (2006). Óp. Cit. p. 41. 
ciudadanía es una llave, un derecho universal y general a participar de la política. Y es que la igualdad, en ningún caso es un derecho natural, es en el más estricto sentido una conquista histórica de las luchas burguesas ${ }^{30}$.

La igualdad es sin duda una forma de enfrentar y de rechazar la dependencia. La discusión de fondo, entonces, gira en torno el contenido de la misma. Si una gran consigna moderna ha sido la lucha por la igualdad (y por la libertad), la pregunta que corresponde hacernos es ¿igualdad de qué?

Sobre esta pregunta (o más correctamente sobre su respuesta) la filosofía jurídica y política contemporánea, ha desarrollado un importante debate. En este punto, es importante, destacar el gesto de John Rawls, ya que con su obra Teoría de la justicia ${ }^{31}$, logró revitalizar la discusión filosófica política. Sus ideas apuntaron a "defender una problemática progresista como la justicia en momentos en que el neoliberalismo hegemónico afirma que la única justicia era la que decretaba el mercado. Esa fue su virtú"32.

No es nuestra intención sumergirnos en el debate igualitarista en esta ocasión. Sin perjuicio de aquello, es importante hacer una pequeña referencia al fondo del debate, ya que es útil para nuestro argumento.

Hoy, nadie podría negar que, al menos consecuencialmente la discusión igualitarista es una disputa respecto de la justicia material. Las posiciones son variadas. Rawls, por ejemplo, apunta por la igualdad de bienes primarios; Dworkin, por la igualdad de recursos, poniendo énfasis en poder hacer frente a la mala suerte bruta; Sen, postula la igualdad de capacidades, poniendo énfasis en los funcionamientos de las personas; Cohen, por su

30 Cfr: Marx, Karl. (2008). La cuestión judía, en: Marx, Karl. 2008. Escritos de juventud sobre el Derecho. Anthropos. España.

31 Cfr: Rawls, John. (1971). A theory of justice. Harvard University Press. EEUU.

32 Borón, Atilio. 2003. "Justicia sin capitalismo, capitalismo sin justicia. Una reflexión acerca de las teorías de John Rawls" en: Análisis político ( $\left.N^{\circ} 49\right)$. Colombia. CLACSO. 
parte sostiene la necesidad de igualar el acceso a las ventajas, etc. Lo indiscutible, es que cualquiera sea la posición filosófica que se siga en este debate, se debe reconocer que todas las teorías de la justicia que entran en la discusión "implican una considerable redistribución de los recursos en la sociedad"33.

En un mundo radicalmente desigual, la discusión sobre la igualdad, sobre su contenido y la necesidad de la redistribución, es un tema central.

Balibar, en perspectiva crítica $^{34}$, ha sostenido que en la lucha respecto de la igualdad y la libertad siempre se genera un rédito entre la expectativa igualitaria y el contenido de esa lucha que es recogido por el sistema jurídico o por la constitución; "un diferencial de insurrección y de constitución, que ninguna representación puramente formal o jurídica de la política puede resumir" 35 . Ese rédito o diferencial, es una característica de las luchas y las reivindicaciones por los derechos asociados a la igualdad. Sostenemos que aquello no es un síntoma, no es un problema tangencial, sino que forma parte de la estructura misma del Derecho.

\section{La contradicción en el Derecho moderno}

La sección anterior ha reflejado nuestro esfuerzo por dar argumentos plausibles que sostengan nuestra primera hipótesis. Las siguientes líneas, responderán a la segunda hipótesis, nos esforzaremos por dar argumentos que permitan sostener que

\footnotetext{
33 Callinicos, Alex. 2006. Óp. Cit. p.109.

34 Balibar no es un pensador dialéctico, por esta razón, denomina paradoja al problema que describe. Desde una perspectiva dialéctica, las paradojas puede ser comprendidas como contradicciones. Y pensar el problema desde esta perspectiva tiene una gran ventaja: las contradicciones a diferencia de las paradojas tienen en su interior la posibilidad de su superación.

Cfr: Ollman, Bertell. 2015. Marxism and the philosophy of internal relations; or, How to replace the mysterious 'paradox' with 'contraditions' that can be studied and resolved. En: Capital \& Class (vol. 39(1)). SAGE: Inglaterra

35 Balibar, Étienne. (2017). Óp. Cít. p. 28-29.
} 
en el Derecho yace una contradicción de carácter fundamental, o dicho de otro modo, el Derecho moderno se configura como una relación social contradictoria.

En párrafos anteriores, argumentamos que el Derecho desde el materialismo histórico puede ser concebido como una relación social históricamente determinada. En su núcleo se esconde su carácter contradictorio.

Ya hemos adelantado algunos de los argumentos que sustentarán nuestra posición. Aquí, nos ocuparemos de estudiar la tensión constante que existe entre la relación básica de producción capitalista (es decir, la relación de explotación), que el Derecho hace posible, y la igualdad como horizonte valorativo del sistema jurídico.

El concepto de contradicción es problemático en bastantes sentidos. En primer lugar, es un término abstracto, que no tiene necesariamente un significado uniforme. Puede referir perfectamente a asuntos relacionados con la lógica aristotélica; a cuestiones propias de la física teórica, tales como la tercera ley de Newton; o hasta a asuntos relacionados con el materialismo histórico como la lucha de clases.

Por lo mismo, este concepto ha sido utilizado en la historia del materialismo histórico con poca uniformidad y poco rigor argumentativo. Sin embargo, el marxismo contemporáneo ha entregado herramientas notables para el estudio de las contradicciones propias de la formación social capitalista.

No es una novedad afirmar que el Derecho nace como una relación social legitimadora de la formación social capitalista, tal y como nos esforzamos por señalar en una sección anterior.

El derecho es la herramienta mediante la que la burguesía naciente pudo dar vida a sus propósitos mercantiles. Pero como sostuvimos, el asunto no es tan simple como sostener que el Derecho beneficia a la clase dominante. El Derecho no es producto de la mala voluntad o la ambición de la clase capitalista, sino que por el contrario, es una de las tantas formas de concreción de los valores liberales-burgueses. Es, también, 
una forma de modificar radicalmente la forma en que se concebía la división social del trabajo. Por lo mismo, es una manera de controlar la división del trabajo social. La relación básica y constituyente de la producción capitalista; la explotación, y la igualdad, están en constante tensión. El Derecho responde concediendo o restringiendo derechos a estos sujetos colectivos históricos, según cómo esta tensión se resuelva.

Bertell Ollman, en sus estudios sobre dialéctica materialista ha entendido la contradicción "como el desarrollo incompatible de diferentes elementos dentro de la misma relación"36. En su reciente obra en torno a las contradicciones del capital, David Harvey sostiene, en una perspectiva muy similar a la de Ollman, que se observa una contradicción cuando existen "dos fuerzas aparentemente opuestas presentes en una situación, una entidad, un proceso o un acontecimiento" 37 .

La igualdad está en el núcleo del Derecho, no porque sea un derecho natural. Al contrario, la igualdad (y particularmente su contenido material), es fruto de la historia, de las luchas de la humanidad. Aquello que consideramos un producto histórico no puede ser considerado natural.

El ideal político normativo que la modernidad ha construido en torno a la idea de igualdad es, en un primer momento, una premisa básica para el desarrollo de las relaciones sociales del capitalismo.

En el acápite anterior sostuvimos que la creación de la categoría de sujetos jurídicos abstractos, es la antesala del intercambio capitalista. Un intercambio se considera equivalente, sólo entre sujetos equivalentes. Adelantamos que el problema de fondo de esta forma de igualdad sin contenido cierto es que desconoce la situación (desigual) concreta de la producción capitalista.

\footnotetext{
36 Ollman, Bertell. (2003). Dance of the Dialectic: Steps in Marx's Method. University of Illinois. EEUU, p. 17.

37 Harvey, David. (2014). Diecisiete contradicciones y el fin del capitalismo. Quito. Traficantes de sueños. p. 17.
} 
Pero si cambiamos el foco del análisis, desde el intercambio hacia la producción capitalista notamos el problema. Y es que cómo ya sostuvimos, la esfera de la producción capitalista necesita de sujetos desiguales, generándose así, una relación en la que un grupo vive del valor que extrae de otro. Este fenómeno es la explotación. Ante esta situación se hace necesario volver a analizar la categoría de sujeto jurídico.

Etimológicamente, entendemos por sujeto de derecho o jurídico, una sustancia o el actor de un acto; otra acepción interesante es aquella proveniente de la palabra latina subjectum, es decir súbdito. De esta forma, el sujeto de derecho es aquel actor en las relaciones que llamamos jurídicas, por un lado; y es aquel que es súbdito del Derecho, por otro.

La clara desigualdad clasista, la pauperización de la clase trabajadora, la exclusión de algunos grupos sociales, han demostrado que la igualdad ante la ley que se ofrece a los sujetos de derecho es insuficiente. Frente a esta situación, el concepto de ciudadanía se vuelve a ser interesante de analizar.

La ciudadanía no es un mero estatus. Tampoco debe reducirse a la mera acción del voto en un sistema representativo. Es una noción compleja que articula aspectos civiles, políticos y sociales, entre otros. Está íntimamente relacionada a la soberanía. Tanto ésta como aquella, son inicialmente actividades insurreccionales o de subversión. La soberanía popular y la ciudadanía son una forma de enfrentar los privilegios y las desigualdades del antiguo régimen. Así, la ciudadanía ve también su fundamento en la noción de igualdad (y en la libertad).

La ciudadanía es, ante todo, una práctica de la política. Es la práctica que hace posible que las luchas y reivindicaciones políticas, tengan recepción jurídica. Son los ciudadanos en su ejercicio soberano quienes constitucionalizan el contenido material de la igualdad. Este proceso de recepción jurídica o de constitucionalización, tal y como señala Balibar, siempre está en deuda con el proceso histórico real. 
Una de las esferas importantes de la ciudadanía, es la ciudadanía social. La noción de ciudadanía social tiene su origen en la obra de T. H. Marshall ${ }^{38}$. Tomando esa perspectiva, Cortina Orts ha defendido que un ciudadano es aquel que

"en una comunidad política goza no sólo de derechos civiles (libertades individuales), en los que insisten las tradiciones liberales, no sólo de derechos políticos (participación política), en los que insisten los republicanos, sino también de derechos sociales (trabajo, educación, vivienda, salud, prestaciones sociales en tiempos de especial vulnerabilidad)"39.

La lucha por la ciudadanía social, es una lucha constante, insurreccional e inacabable. Pero ocurre que la lucha igualitaria y por la libertad, propias de esta esfera de la ciudadanía, ha desviado el foco de las luchas obreras.

La lucha ciudadana no es una lucha por la erradicación de las relaciones y las condiciones que hacen posible la producción capitalista, sino por la generación de nuevas condiciones en la reproducción capitalista. Esto no significa que la ciudadanía social tenga un carácter meramente asistencial, muy por el contrario, su esencia es universalista y profundamente igualitarista, pero la perspectiva histórico-materialista permite sostener que ha olvidado la explotación como problema central respecto de la (des)igualdad.

A estas alturas, nuestra posición central puede observarse más prístinamente. Hemos sostenido que el derecho moderno se configura como una relación contradictoria, ya que al momento de poner en marcha la estructura jurídica, por un lado, los individuos son representados como sujetos jurídicos iguales formalmente habilitándolos así para intercambiar mercancías (incluyendo la fuerza de trabajo como mercancía), pero fuera

38 Cfr: Marshall, Thomas H. (1998). Ciudadanía y clase social. Alianza Editorial. España.

39 Cortina, Adela. (2001). Ciudadanos del mundo: Hacia una teoría de la ciudadanía. Madrid. Alianza. p. 66. 
de la dimensión del intercambio, en la esfera de la producción, hemos señalado que los sujetos son radicalmente desiguales. Para la producción de mercancías, que tienen como fin ser intercambiadas, es necesario que un sector de la humanidad sea despojado del acceso a los medios de producción.

En este sentido, el Derecho considerando a los sujetos iguales en abstracto, otorga las condiciones para que sea posible la producción y, por ende, el intercambio capitalista. No importa la clase a lo que un individuo pertenezca, el Derecho lo considerará igual a aquel que en términos de poder es profundamente desigual.

Por otro lado, en la lucha igualitaria por mejorar las condiciones de vida de la mayor parte de la humanidad, el Derecho convierte a los individuos en ciudadanos. Gracias a esta noción, la igualdad actualiza constantemente su contenido material, conforme a cómo se actualizan las demandas y las luchas históricas, mejorando así las condiciones de reproducción capitalista. Es por esa razón que el Derecho moderno se configura como una relación social contradictoria ya que en él hay dos fuerzas opuestas, dos intereses opuestos respecto del producto social. Dicha contradicción permite que el contenido igualitario del derecho avance de forma dinámica ${ }^{40}$.

Por un lado se hace legal la explotación y por otro trata de hacer frente a la miseria que ésta acarrea. El Derecho crea el panorama normativo que hace posible el intercambio y la explotación capitalista; pero a su vez ofrece un horizonte valorativo igualitario asociado a la ciudadanía que busca remediar las desigualdades relacionadas a la producción capitalista.

Visto desde esta perspectiva, el Derecho se posicionara por sobre las clases. Al resolver un conflicto en algún momento

\footnotetext{
40 Una tesis similar ha sido desarrollada por Duncan Kennedy, quien ha sostenido que en el sistema jurídico liberal existe una contradicción interna fundamental ente individualismo y altruismo.

Cfr: Kennedy, Duncan. (1976). "Form and Substance in Private Law Adjudication", en: Harvard Law Review. EEUU. Universidad de Harvard.
} 
histórico beneficiará a la clase dominante (por ejemplo, al momento en que el derecho de propiedad se vuelve un derecho fundamental) y en otro a la clase trabajadora (cuando se le concede derecho a huelga, por ejemplo).

El Derecho (y obviamente también el Estado) se vuelve, en la lectura que hace Iñigo Carrera

"el representante del capital total de la sociedad. Tiene a su cargo la unidad del movimiento de la acumulación de los capitales individuales. Los capitalistas individuales se encargan de explotar a sus obreros, el Estado es el explotador de la clase obrera como tal. Su función es explotar, hacer que la clase obrera produzca plusvalía, pero que lo haga en condiciones normales" ${ }^{\prime 1}$

Toda victoria de la clase explotada, es en rigor, una victoria para la formación social capitalista ya que la legitima. Ese horizonte de igualdad, al que las luchas sociales aspiran mediante la consagración de derechos sociales, se aleja cada vez que se consagran derechos en la lógica burguesa.

\section{A modo de colofón}

La racionalidad del Derecho moderno, ha sido la racionalidad del derecho igual, tal y como Marx lo señaló más de una vez. En ese escenario, la tradición del materialismo histórico ha sido firme en evidenciar la desigualdad estructural que exististe en la sociedad moderna. Tal desigualdad se hace evidente cuando lo que se pone en el foco del análisis es la relación o la categoría clase social.

La igualdad abstracta y formal es la base de la competencia capitalista. El movimiento del capital hace incompatibles los intereses de la clase que posee y controla los medios de producción con los intereses de la clase trabajadora. Más aún, la lógica de la ganancia hace que cada individuo sea considerado

41 Carrera, Juan Íñigo. Óp. Cit. p. 61 
como competencia para otro que tenga sus mismos propósitos, incluso dentro de su misma clase.

Precisamente, ateniendo a la racionalidad competitiva del capital, Carlos de Cabo ha concluido que la solidaridad no tiene cabida en el sistema constitucional liberal, "sino que la insolidaridad es un principio constitucional necesario del sistema liberal. Precisamente porque es el constitucionalismo formal, del universalismo, de la abstracción, del sujeto (individual), en definitiva y paradójicamente «el constitucionalismo de la igualdad ${ }^{42}$.

El punto que destaca el profesor de Cabo no es menor. La solidaridad no debe ser omitida al momento de analizar el espectro valorativo de la modernidad. Ese horizonte de igualdad y libertad que la modernidad levantó como bandera de lucha está íntimamente relacionado con la solidaridad, sobre todo cuando de lo que se trata es de las reivindicaciones de la clase trabajadora.

En este sentido una interesante definición de la solidaridad, profundamente arraigada en el materialismo histórico, es ofrecida por Michael Albert. Para él, "la solidaridad es una noción muy básica que se preocupa por el bienestar de los demás, en lugar de obstaculizar su desarrollo. Cuanto mayor sea el grado de solidaridad, más óptimo será nuestro bienestar" ${ }^{\prime 3}$.

Es muy interesante notar que el horizonte valorativo moderno va a contrapelo de las prácticas efectivas del capital. Sobre este problema, Marx puede aportar algunas luces.

Es cierto que Marx nunca ofreció una solución clara a los problemas del capitalismo, su principal tarea fue describir el movimiento del mismo y evidenciar sus problemas y contradicciones. Aun así, en su texto Crítica al programa de Gotha, Marx

42 De Cabo Martín, Carlos. (2006). Teoría constitucional de la solidaridad. Marcial Pons. Madrid. p. 44.

43 Albert, Michael. (2003). "Economía participativa". En: Conferencia en Woods Hole. Woods Hole, Estados Unidos. 8 p. p. 1. 
pone en el centro de su análisis a la igualdad, a la libertad y a la solidaridad, con el objeto de dar luces de cómo superar la formación social capitalista.

En el mencionado texto, Marx recuerda al movimiento obrero que el núcleo de las luchas de los trabajadores debe estar puesto en recuperar el control de la producción (sin descuidar la esfera de la reproducción). Para lograr aquello propone (pensando en una sociedad que ha superado las contradicciones de clase), un principio igualitario de derecho igual (es decir un principio de racionalidad burguesa) en el que cada uno recibe según su contribución al producto social.

A este principio, siguiendo a Fernando Lizarraga, puede incorporársele un criterio diferenciador. De esta forma, el principio quedaría esbozado de esta manera: "de cada quien según su capacidad a cada quien proporcionalmente a su contribución laboral, después de que se han separado fondos para satisfacer necesidades económicas y necesidades comunes" ${ }^{44}$, aceptando sólo las desigualdades que vengan en beneficio de todos.

Aquel, es un principio de transición entre el capitalismo y una forma distinta de organización del producto social, democrática, igualitaria y solidaria, dónde aún puede ser aplicable el derecho en la lógica moderna y burguesa, los individuos son tratados como sujetos de derecho pero, ahora, se consideran sus condiciones materiales.

Yendo más allá, para aquel momento en que la lógica de la producción capitalista se ha superado totalmente, Marx propone el principio de necesidad:

"En la fase superior de la sociedad comunista, cuando haya desaparecido la subordinación esclavizadora de los individuos a la división del trabajo, y con ella, la oposición entre el trabajo intelectual y el trabajo manual; cuando el trabajo no sea solamente un medio de vida, sino la primera necesidad vital; cuando, con el desarrollo

44 Lizarraga, Fernando. (2011). El marxismo y la justicia social. Santiago. Nexos, p. 99. 
de los individuos en todos sus aspectos, crezcan también las fuerzas productivas y corran a chorro lleno los manantiales de la riqueza colectiva, sólo entonces podrá rebasarse totalmente el estrecho horizonte del derecho burgués, y la sociedad podrá escribir en su bandera: ¡De cada cual, según sus capacidades; a cada cual, según sus necesidades!" ${ }^{45}$.

El principio de necesidad es un principio igualitario complejo, que pone su atención no en lo cuantitativo sino en lo cualitativo. Es un principio de igualdad diferenciada y profundamente solidario. Tal y como el mismo Marx subraya, este principio sólo es aplicable cuando el modo de producción, la formación social completa, sea reemplazada por una forma realmente igualitaria, democrática y participativa de ordenar y producir la vida que permita a los individuos operar bajo una racionalidad basada no en la competencia, sino que en la cooperación. Para Marx la humanidad es igual en su género pero diferente en su individualidad, por lo mismo sostiene que "una realidad universal basada en la desigualdad reclama derechos desiguales" 46

Dicho de otro modo, Marx ofrece herramientas para pensar un horizonte que supere o resuelva la contradicción que hemos intentado exponer en estas páginas. Desde la perspectiva que ofrece el filósofo de Tréveris, los valores propios de la modernidad, sólo son alcanzable solamente más allá de las fronteras de la formación social capitalista.

En últimos términos, para Marx, el logro del horizonte valorativo moderno implica, necesariamente, superar la formación social actual, y por ende la forma jurídica burguesa moderna. De modo tal que la igualdad debe poner su foco de análisis en la producción y no sólo en la reproducción.

En cierto modo, la igualdad, la libertad y sobre todo la solidaridad ofrecen un panorama para superar la contradicción

\footnotetext{
45 Marx, Karl. (2000). Crítica al programa de Gotha. El aleph. Buenos Aires. p. 17.

46 Marx, Karl. (1982). Escritos de juventud. EFE. México, p. 253.
} 
inmanente al sistema jurídico. "En formaciones sociales configuradas con arreglo a otro modo de producción, la solidaridad como principio constitucional tendría unas características bien distintas: sería precisamente el elemento estructurante y no el corrector del sistema" ${ }^{\prime 4}$.

Hay, pues, todo un contenido nuevo que justifica que el Derecho, en esa nueva sociedad que muy difuminadamente imaginó Marx, en aquella asociación de individuos libres que pensó, "no pueda ser considerado como derecho burgués, y que por el contrario pueda hablarse de un nuevo derecho en cuanto que respondiendo, sobre todo, a las nuevas condiciones materiales de producción (propiedad civil) se requieren nuevas relaciones jurídicas" ${ }^{48}$.

En la contradicción que yace en el núcleo del derecho moderno, está contenido el germen de la superación de la propia forma jurídica.

\section{Bibliografía}

Albert, Michael. 2003. Economía participativa. En: Conferencia en

Woods Hole. Woods Hole. Estados Unidos. 8 p. p. 1.

Alegre, Marcelo. 2010. Igualdad, Derecho y Política. Buenos Aires. Fontamara. P. 13

Balibar, Étienne. 2017. La igualibertad. España. Herder. p.180.

Borón, Atilio. 2003. "Justicia sin capitalismo, capitalismo sin justicia. Una reflexión acerca de las teorías de John Rawls" en: Análisis político ( $\left.\mathrm{N}^{\circ} 49\right)$. Colombia. CLACSO.

Callinicos, Alex. 2006. Igualdad. Siglo XXI. España.

Callinicos, Alex. 2011. Contra el posmodernismo. Buenos Aires. Ediciones RyR.

Capella, Juan Ramón. 2008. Fruta prohibida: una aproximación histórico-teorética del derecho y del Estado. España. Trotta.

47 De Cabo, Carlos. Óp. Cit. p. 107.

48 Sánchez Vázquez, Adolfo. (1976). "Pashukanis, teórico marxista del derecho", en: Pashukanis, E. B. 1976. La teoría general del Derecho y el marxismo. Barcelona, Grijalbo. p. 115. 
Cárcova, Carlos. (2012). Las Teorías Jurídicas Post Positivistas. Buenos Aires. Abeledo-Perrot

Carrera, Juan Íñigo. 2012. "El capital: determinación económica y subjetividad política": en: Crítica Jurídica ( $\left.{ }^{\circ} 34\right)$. UNAM: México

Cortina Adela. 2001. Ciudadanos del mundo: Hacia una teoría de la ciudadanía. Madrid. Alianza.

De Cabo Martín, Carlos. 2006. Teoría constitucional de la solidaridad. Marcial Pons. Madrid.

Kelsen, Hans. 2010. "La función de la constitución", en: Revista del Instituto de investigación Ambrosio L. Gioja (año IV, $\mathrm{n}^{\circ}$ 5). Pp. 150-157.

Kennedy, Duncan. 1976. "Form and Substance in Private Law Adjudication" En: Harvard Law Review. EEUU. Universidad de Harvard.

Lizarraga, Fernando. 2011. El marxismo y la justicia social. Santiago. Nexos.

Macía Manso, Ramón. 2012. Cientificismo. Modalidades. Consecuencias. Oviedo, España. Ediciones de la U. de Oviedo.

Marshall, Thomas H. 1998. Ciudadanía y clase social. Alianza Editorial. España.

Marx, Karl. 2000. Crítica al programa de Gotha. El aleph. Buenos Aires.

Marx, Karl. 2010. El capital: Crítica de la economía política (T1). Santiago de Chile. LOM.

Marx, Karl. 1982. Escritos de juventud. EFE. México.

Marx, Karl. 1980. Historia crítica de la Teoría de la Plusvalía (T1). FCE. México.

Marx, Karl. 2008. La cuestión judía, en: Marx, Karl. 2008. Escritos de juventud sobre el Derecho. Anthropos. España.

Marx, Karl. 1971. Manifiesto comunista. Editorial Universitaria. Santiago.

Mieville, China. 2005. Between equal rights: A marxist theory of Internacional Law. Brill: Oxford. Inglaterra.

Ollman, Bertell. 2003. The dance of dialectics. Steps in Marx' method. University of Illinois press, Urbana and Chicago. EEUU 
Ollman, Bertell. 2015. Marxism and the philosophy of internal relations; or, How to replace the mysterious 'paradox' with 'contraditions' that can be studied and resolved. En: Capital \& Class (vol. 39(1)). SAGE: Inglaterra

Osorio, Jaime. 2014. Estado, reproducción del capital y lucha de clases: la unidad económico/política del capital. U. Autónoma de México - Instituto de investigaciones económicas -CLACSO. México.

Pashukanis, E. B. 1976. La teoría general del Derecho y el marxismo. Barcelona, Grijalbo.

Pérez Luño, Antonio. 2007. Dimensiones de la igualdad. Cuadernos "Bartolomé de las Casas" (núm. 34). Dykinson. Madrid.

Pérez Soto, Carlos. 2001. Para una crítica del poder burocrático: comunistas otra vez. Santiago de Chile. LOM-U. ARCIS

Rancière, Jacques. 2007. El método de la igualdad, conferencia en Cerisy-La Salle. Francia

Rawls, John. 1971. A theory of justice. Harvard University Press. EEUU.

Rivera Lugo, Carlos. 2017. "Comunismo y Derecho: reflexiones sobre la crisis actual de la forma jurídica”, en Correas, Oscar y Wolkmer, Antonio. 2017. La teoría crítica del Derecho en América latina. España. Akal.

Ruiz, Alicia E. C. 2000. Idas y vueltas: por una teoría crítica del Derecho. Buenos Aires. UBA.

Sacristán, Manuel. 1984. "De la idealidad en el Derecho". En: Sacristán, Manuel. 1984. Papeles de filosofía. Panfletos y materiales II. Icaria Antrazyt. España.

Sánchez Vázquez, Adolfo. 1976. "Pashukanis, teórico marxista del derecho”, en: Pashukanis, E. B. 1976. La teoría general del Derecho y el marxismo. Barcelona, Grijalbo. 